Hongyan Li, Cong Sun, Ruyi Li, Yongqiang Fu, Limeng Song, Aiwu Yang and Hongli Liu*

\title{
Preparation and characterization of silica aerogel/ polymethyl methacrylate composites with electrostatic interaction phase interface
}

https://doi.org/10.1515/polyeng-2017-0402

Received November 17, 2017; accepted February 4, 2018; previously published online August 1, 2018

Abstract: Silica aerogel (SA)/polymethyl methacrylate (PMMA) composites were prepared through electrostatic interaction, which could form phase interface. The effects of electrostatic interaction phase interface on the performance of the composites were studied. The SA/polymer hybrid composites were prepared because of the negative charges in SA and the positive charges in polymer. Characterizations of the SA, polymer, and composites were performed using Fourier transform infrared spectroscopy, scanning electron microscopy, transmittance, gel permeation chromatography, differential scanning calorimetry, zeta potential, and so on. The results indicated that SA has negative charges, whereas the polymer has positive charges; thus, the polymer was absorbed on the surface of the SA skeleton to form an electrostatic interaction phase interface. The composites achieved better transmittance than that of SA/PMMA with the electrostatic interaction phase interface.

Keywords: electrostatic interaction; phase interface; PMMA; silica aerogel.

\footnotetext{
*Corresponding author: Hongli Liu, School of Materials Science and Engineering, Tianjin Chengjian University, Tianjin 300384, P. R. China; and Tianjin Key Laboratory of Soft Soil Characteristics and Engineering Environment, Tianjin 300384, P. R. China, e-mail: Ihlbh@163.com
}

Hongyan Li: School of Materials Science and Engineering, Tianjin Chengjian University, Tianjin 300384, P. R. China; and Tianjin Key Laboratory of Soft Soil Characteristics and Engineering Environment, Tianjin 300384, P. R. China

Cong Sun, Ruyi Li, Yongqiang Fu and Limeng Song: School of Materials Science and Engineering, Tianjin Chengjian University, Tianjin 300384, P. R. China

Aiwu Yang: Tianjin Key Laboratory of Soft Soil Characteristics and Engineering Environment, Tianjin 300384, P. R. China; and School of Civil Engineering, Tianjin Chengjian University, Tianjin 300384, P. R. China

\section{Introduction}

Silica aerogels (SA) are nanoporous amorphous materials prepared using the sol-gel technique and supercritical drying [1], featuring high porosity, low density, high specific surface area, and ultralow thermal conductivity at an ambient temperature [2-5]. The ultrahigh porosity of aerogels and their porous network structure render the bulk material easy to break, therefore, limiting their excellent performance [6]. Thus, different types of fibers are generally doped into the aerogels to reinforce their mechanical properties [7-10].

Some mechanical properties of SA were reinforced by different strategies. It can be observed that the mechanical properties of SA could be improved by adding fibers or particles in the initial sol [11-14]. However, this method has some shortcomings. The short fibers or powders, which are used as reinforcement, are normally incorporated by mechanical stirring; hence, these powders inevitably exhibit nonuniform dispersion in the aerogels, which leads to a deterioration of the performance of the aerogels. As for fiber felt reinforcement, different shrinkage rates between the fibers and aerogel matrices would lead to large residual stresses, which could lead to large cracks. These cracks may be detrimental for aerogels that are to be used as thermal insulators, as they may increase the gaseous thermal conductivity. In addition, large cracks may decrease the mechanical strength of composites [15]. Compared with the method of secondary reinforcement, the enhanced effect of polymer cross-linking on the mechanical properties of aerogels is very impressive [16-19]. Scherer et al. [20, 21] used an organic solvent (precursor solution, organic solvent, or their mixture) to soak and wash a wet gel several times, and this method reinforced the strength of the solid nanoparticle contact points and facilitated additional condensation reactions. Moreover, the addition of an organic polymer into an aerogel can result in cross-linking with the reactive groups present on the aerogel surface [22]. For example, a silica-based aerogel was chemically modified with amine or epoxy groups to allow cross-linking with other organic polymers, thus significantly increasing the aerogel strength [23]. 
Copolymerization refers to the reaction of two or more compounds polymerizing into a substance under certain conditions. According to the different molecular structures of the polymer, polymerization methods could be divided into random copolymerization [24], block copolymerization [25], alternating copolymerization [26], or graft copolymerization [27].

We have studied load transfer across the electrostatic interaction phase interfaces in a previous article [28], the covalent bonding phase interface, van der Waals, and electrostatic interaction phase interface are the media for load transfer in composites, respectively. The electrostatic interaction phase interface has high interfacial thermal resistance, which is the physical interaction between the silica phase and the polymer phase, and composites with the electrostatic interaction phase interface have good thermal insulation properties. In this research, we further investigated the construction of phase interface, and the effects of the contents of methacryloxyethyltrimethyl ammonium chloride (MTC) on the optical performance of composites.

\section{Materials and methods}

\subsection{Materials}

Methyl methacrylate (MMA) and MTC were purchased from Sigma-Aldrich, WI, USA. Benzoyl peroxide (analytical grade) was supplied by Aladdin Industrial Corporation (Shanghai, China). Tetraethoxysilane and hydrochloric acid were obtained from Tianjin Chemical Reagent Corporation (China). Ammonium hydroxide was purchased from Beijing Chemical Factory (China). The other reagents were all analytical grade.

\subsection{Preparation of SA}

The silica wet gels were synthesized from mixtures of tetraethoxysilane, deionized water $\left(\mathrm{H}_{2} \mathrm{O}\right)$, and ethanol. After $15 \mathrm{~min}$ of stirring, hydrochloric acid $(0.1 \mathrm{~mol} / \mathrm{l})$ was added to the mixture dropwise until the hydrochloric acid/tetraethoxysilane molar ratio reached 0.0018. After another $2 \mathrm{~h}$ of stirring, ammonium hydroxide $\left(\mathrm{NH}_{4} \mathrm{OH}\right)$ $(0.05 \mathrm{~mol} / \mathrm{l})$ was added dropwise to adjust the solution to $\mathrm{pH}$ 5.5-6.0. The solution was then left for silicate polymerization and aging for $24 \mathrm{~h}$, forming the silica wet gels. After being soaked in ethanol for 5 days, the ethanol in the wet gels was removed by supercritical carbon dioxide drying $\left(\mathrm{P}_{\mathrm{C}}=10 \mathrm{MPa}\right.$ and $\left.\mathrm{T}_{\mathrm{C}}=318.15 \mathrm{~K}\right)$, resulting in the formation of the corresponding aerogels.

\subsection{Preparation of composites}

The amounts of MTC were changed from 2 to 10 weight accounts relative to total monomer used in the polymerizations. Before the polymerization process, 100 weight account of MMA and benzoyl peroxide were dispersed in $50 \mathrm{ml}$ of distilled water and stirred with magnetic stirring. The polymerizations were carried out with a thermometer and a magnetic stirrer. Then, the MTC was added to this solution, followed by ultrasonic dispersion for $30 \mathrm{~min}$ at room temperature. The MTC contents are 2, 4, 6, 8, and 10 weight accounts, respectively. The SA was added to the mixed solution, and then followed by ultrasonic dispersion for $1.5 \mathrm{~h}$. The uniformly dispersed mixture was polymerized in a water bath, the temperature and the stirring rate were increased to $60^{\circ} \mathrm{C}$ and $220 \mathrm{rpm}$, respectively, and the reaction was continued for 24-48 $\mathrm{h}$ under these conditions to increase the conversion of the monomers. Lastly, the mixed solution was cooled to room temperature. The composites were named as SA/P(MMA-co-2\%MTC), SA/P(MMA-co4\%MTC), SA/P(MMA-со-6\%MTC), SA/P(MMA-со-8\%MTC), and SA/P(MMA-co-10\%MTC) according to the 2, 4, 6, 8, and 10 weight accounts of the MTC contents, respectively. Through the addition of $\gamma$-methacryoxypropytrimethoxys ilane (KH570), polymethyl methacrylate (PMMA)-KH570SA was prepared as a control group. The compositions and polymerization reactions of the samples are shown in Table 1 and Scheme 1. The sample numbers in the following discussion section are based on Table 1.

Table 1: Composition.

\begin{tabular}{llrrrr}
\hline No & Sample & $\begin{array}{r}\text { SA } \\
\text { (wt\%) }\end{array}$ & & \multicolumn{3}{c}{ Reaction ratio } \\
\cline { 4 - 6 } & & 100 & 0 & 0 & 0 \\
\hline A & SA & 0 & 100 & 0 & 0 \\
B & PMMA & 0 & 100 & 7 & 0 \\
C & P(MMA-co-MTC) & 10 & 100 & 0 & KH570 \\
D & SA/PMMA & 10 & 100 & 7 & 7 \\
E & PMMA-KH570-SA & 10 & 100 & 7 & 0 \\
F & SA/P(MMA-co-MTC) & 10 & 100 & 2 & 0 \\
G & SA/P(MMA-c0-2\%MTC) & 10 & 100 & 4 & 0 \\
H & SA/P(MMA-co-4\%MTC) & 10 & 100 & 6 & 0 \\
I & SA/P(MMA-c0-6\%MTC) & 10 & 100 & 8 & 0 \\
J & SA/P(MMA-c0-8\%MTC) & 10 & 100 & 0 \\
K & SA/P(MMA-co-10\%MTC) & 10 & 100 & 10 & 0 \\
L & SA-MTC & 10 & 0 & 7 & 0 \\
M & 2MTC-98MMA & 0 & 98 & 2 & 0 \\
N & MTC & 0 & 0 & 7 & 0 \\
\hline
\end{tabular}




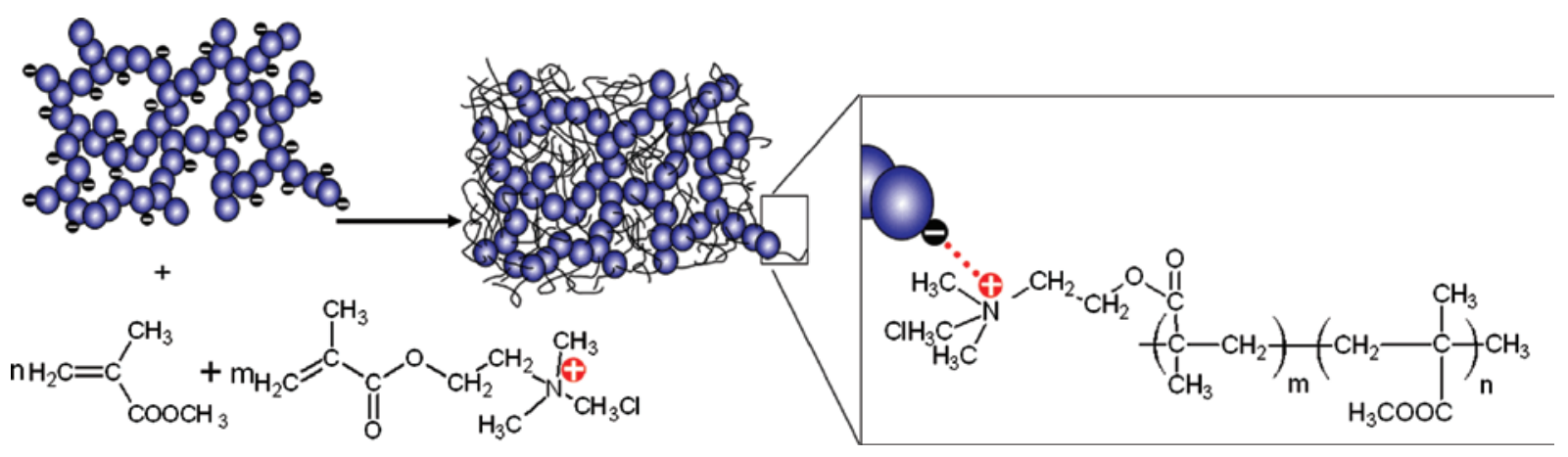

Scheme 1: Preparation of SA/P(MMA-co-MTC) composite by electrostatic interaction.

\subsection{Characterization}

The Fourier transform infrared (FTIR) spectroscopy used in this study was a Nicolet 6700 model with a spectrum range of $4000-400 \mathrm{~cm}^{-1}$. During the measurement, sample powders were diluted in $\mathrm{KBr}$ ( $\mathrm{KBr}$ is transparent to infrared radiation). Afterward, those powders were pressed into pellets because it is very difficult to produce a slab sample that is thin enough to transmit infrared radiation within the measured wavelengths. A zeta potential analyzer (Malvern Zetasizer Nano series, UK) was used to measure the zeta potential values of the samples. The morphology of specimens was observed using Philips XL30 scanning electronic microscopy (Eindhoven, The Netherlands). The molecular weight and molecular weight distribution of the copolymers were measured using the TDA305 multidetection gel chromatograph. With tetrahydrofuran as a solvent, weighed $0.005 \mathrm{~g}$ test substance, dubbed $0.001 \mathrm{~g} / \mathrm{ml}$ solution, and a nylon membrane was used after the filtration test. Haze measurements were carried out according to the ASTM 1003-92 standard, using an integrating sphere attached to the spectrophotometer. The samples' refractive indexes were measured with the WYA-ZL Abbe refractometer prepared by the Shanghai Physical Optical Instrument Factory. A differential scanning calorimeter (DSC; Maia200 F3, Netzsch, Germany) was used to determine the glass transition temperature ( $\mathrm{Tg}$ ) of the synthesized composites. The samples were heated to $100^{\circ} \mathrm{C}$ and left at that temperature for $3 \mathrm{~min}$, and then cooled to $-50^{\circ} \mathrm{C}$ under a nitrogen atmosphere and reheated to $100^{\circ} \mathrm{C}$. The rate of

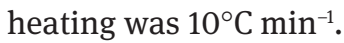

\section{Results and discussion}

Figure 1 shows the FTIR spectra of (A) SA, (B) PMMA, (C) SA/P(MMA-co-MTC), (D) SA/PMMA, (E) PMMA-KH570-SA,

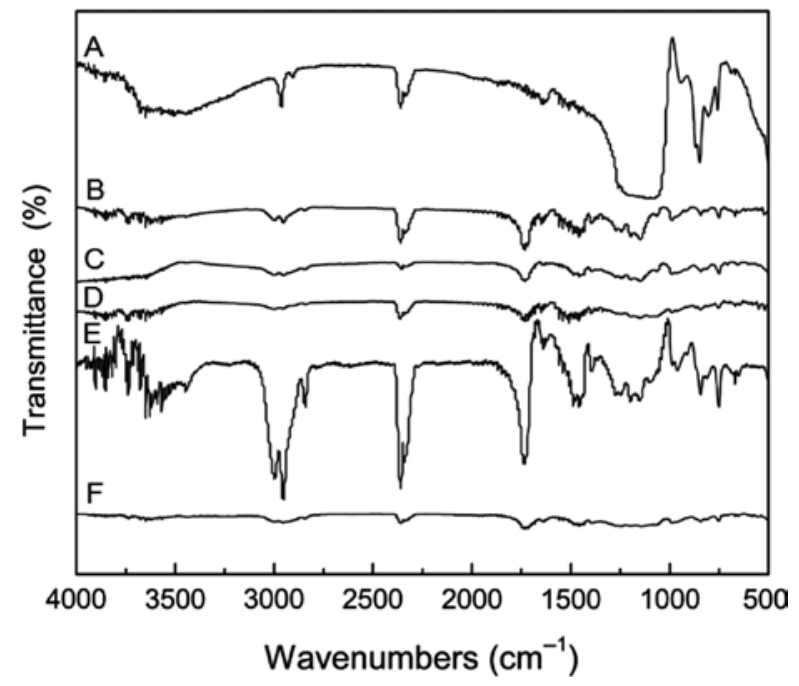

Figure 1: FTIR spectra of (A) SA, (B) PMMA, (C) P(MMA-co-MTC), (D) SA/PMMA, (E) PMMA-KH570-SA, and (F) SA/P(MMA-Co-MTC)

and (F) P(MMA-co-MTC). The absorption bands of curve A at approximately $3436 \mathrm{~cm}^{-1}, 1636 \mathrm{~cm}^{-1}$, and $1096 \mathrm{~cm}^{-1}$ are relative to the stretching vibrations of $\mathrm{Si}-\mathrm{OH}, \mathrm{H}-\mathrm{O}-\mathrm{H}$, and $\mathrm{Si}-\mathrm{O}-\mathrm{Si}$, respectively. The bands at approximately $3500 \mathrm{~cm}^{-1}$ and $1630 \mathrm{~cm}^{-1}$ are ascribed to the residual water. Curve B is the FTIR spectrum of PMMA, the absorption peak at approximately $2960 \mathrm{~cm}^{-1}$ is relative to the stretching vibration of $\mathrm{sp}^{3} \mathrm{C}-\mathrm{H}$ bond, the vibration of the $\mathrm{C}=\mathrm{O}$ can be found at approximately $1730 \mathrm{~cm}^{-1}$. Curve D is the FTIR spectrum of SA/PMMA, the absorption band at approximately $3436 \mathrm{~cm}^{-1}$ is relative to the stretching vibration of $\mathrm{Si}-\mathrm{OH}$, the absorption peak at approximately $1636 \mathrm{~cm}^{-1}$ bending vibration caused by $\mathrm{H}-\mathrm{O}-\mathrm{H}$, and the absorption peak at approximately $1096 \mathrm{~cm}^{-1}$ corresponds to the antistretching vibration of Si-O-Si. Some absorption bands of PMMA were found in curve D. Curve $\mathrm{E}$ is the FTIR spectrum of PMMA-KH570-SA. The absorption band at approximately $1510 \mathrm{~cm}^{-1}$ and $2900 \mathrm{~cm}^{-1}$ are relative to the stretching vibration of $\mathrm{C}-\mathrm{C}$ and $-\mathrm{CH}_{3}$, respectively. 
There is a characteristic peak caused by $\mathrm{Si}-\mathrm{C}$ at approximately $750 \mathrm{~cm}^{-1}$, which indicated that the PMMA-KH570$\mathrm{SA}$ was present. The curves $\mathrm{C}$ and $\mathrm{F}$ are the FTIR spectra of SA/P(MMA-co-MTC) and P(MMA-co-MTC), respectively. The absorption band at approximately $1510 \mathrm{~cm}^{-1}$ stretching caused by C-C. The absorption peak at approximately $2360 \mathrm{~cm}^{-1}$ is relative to the stretching vibration of $-\mathrm{NH}_{2}^{+}$. The presence of SA/P(MMA-co-MTC) and P(MMA-co-MTC) could be demonstrated.

As shown in Table 2, zeta potential values of SA, MTC, and PMMA are $-38.61 \mathrm{mV}, 54.63 \mathrm{mV}$, and $0 \mathrm{mV}$, respectively. The zeta potential value of SA is a negative value. It could be attributed to the water absorbed on the SA surface, which would ionize hydroxyl; therefore, it is the negative value in the zeta potential. The MTC could ionize an unsaturated ethylene group and a quaternary ammonium salt group, so the zeta potential value of MTC is a positive value. The zeta potential value of SA-MTC polymer is $1.2 \mathrm{mV}$. The negative charges carried by SA and the positive charges carried by the MTC were neutralized, so the amounts of charge by SA-MTC lie between those of SA and MTC. The zeta potential value of 2MTC98MMA polymer is $15.65 \mathrm{mV}$, which indicated that MTC

Table 2: Zeta potential results and DSC results of samples.

\begin{tabular}{llrr}
\hline No & Samples & Zeta potential $(\mathrm{mV})$ & $\mathrm{Tg}\left({ }^{\circ} \mathrm{C}\right)$ \\
\hline A & SA & -38.61 & - \\
B & PMMA & 0 & 113 \\
N & MTC & 54.63 & - \\
L & SA-MTC & 1.2 & - \\
M & 2MTC-98MMA & 15.65 & - \\
D & SA/PMMA & -22.35 & 116 \\
G & SA/P(MMA-co-2\%MTC) & -19.35 & 99 \\
H & SA/P(MMA-co-4\%MTC) & -17.95 & 101 \\
I & SA/P(MMA-co-6\%MTC) & -10.65 & 107 \\
J & SA/P(MMA-co-8\%MTC) & -2.36 & 109 \\
K & SA/P(MMA-co-10\%MTC) & 1.67 & 109 \\
\hline
\end{tabular}

monomer was absorbed into MMA molecular chain. The zeta potential value of 2MTC-98MMA polymer is much smaller than that of the MTC. The reason was that only 2\% MTC of 2MTC-98MMA polymer was contained in the solution, and thus the amounts of charge were small and the potential value of 2MTC-98MMA was much lower compared with that of the MTC solution. The zeta potential value of SA/PMMA is $-22.35 \mathrm{mV}$. Because the zeta potential value of PMMA is $0 \mathrm{mV}$, the zeta potential value of SA is $-38.61 \mathrm{mV}$, and the charges could be neutralized. Data from D, G, H, I, J, and K, zeta potential values were $-22.35 \mathrm{mV},-19.36 \mathrm{mV},-17.95 \mathrm{mV},-10.65 \mathrm{mV},-2.36 \mathrm{mV}$, and $1.67 \mathrm{mV}$, respectively. In these six groups, $\mathrm{SA}$ is invariant, PMMA have almost no charges, and only the MTC has charges. With the increase of MTC, the positive charges of composite gradually increased, so the charges of composite gradually moved to a positive number. When the MTC contents reached $10 \%$, MTC could neutralize the negative charges of the SA surface, the zeta potential of the composite was not charged, and thus the MTC charged is equal to the amount of negative charges of SA/P(MMA-co$10 \%$ MTC) on the surface.

The scanning electron microscopy (SEM) photographs of (A) SA, and (B) SA/P(MMA-co-MTC) are shown in Figure 2. As shown in Figure 2A, the bright fields are silicon dioxide primary particles, and secondary particles are deposited into a random three-dimensional network structure. The dark field is the pore in the SA structure. SA forms particles with an average diameter of approximately 10-20 nm, which could be arranged with narrow size distribution.

As shown in Figure 2B, the bright fields are composite particles, the dark field is the pore in the composite structure. The composite particles' size distribution and the pore size are almost similar with that of SA. SA forms a three-dimensional network structure. SA has negative charges, and the polymer has positive charges, which
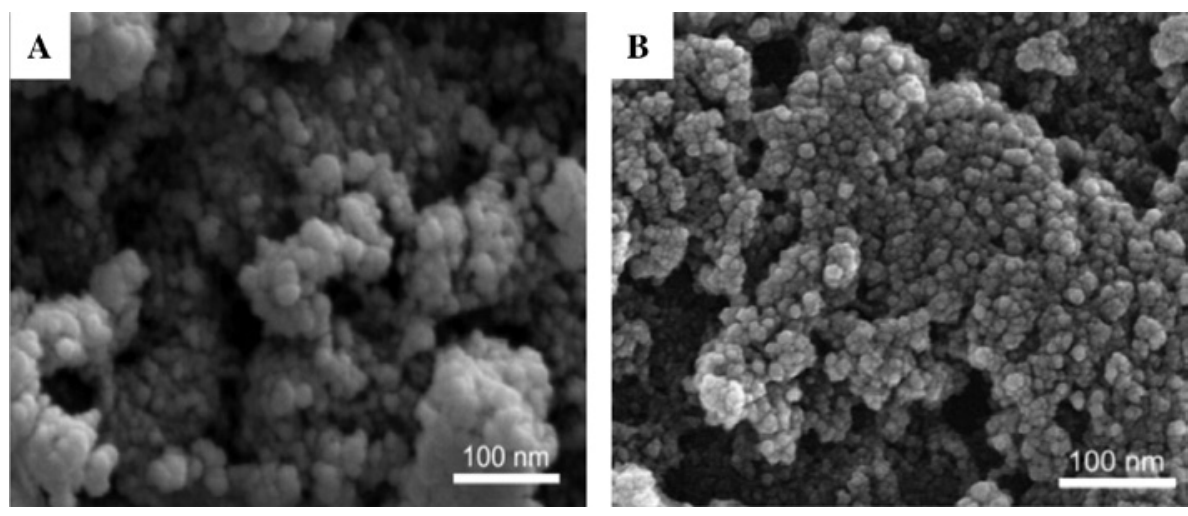

Figure 2: SEM images of (A) SA and (B) SA/P(MMA-co-MTC) composite. 


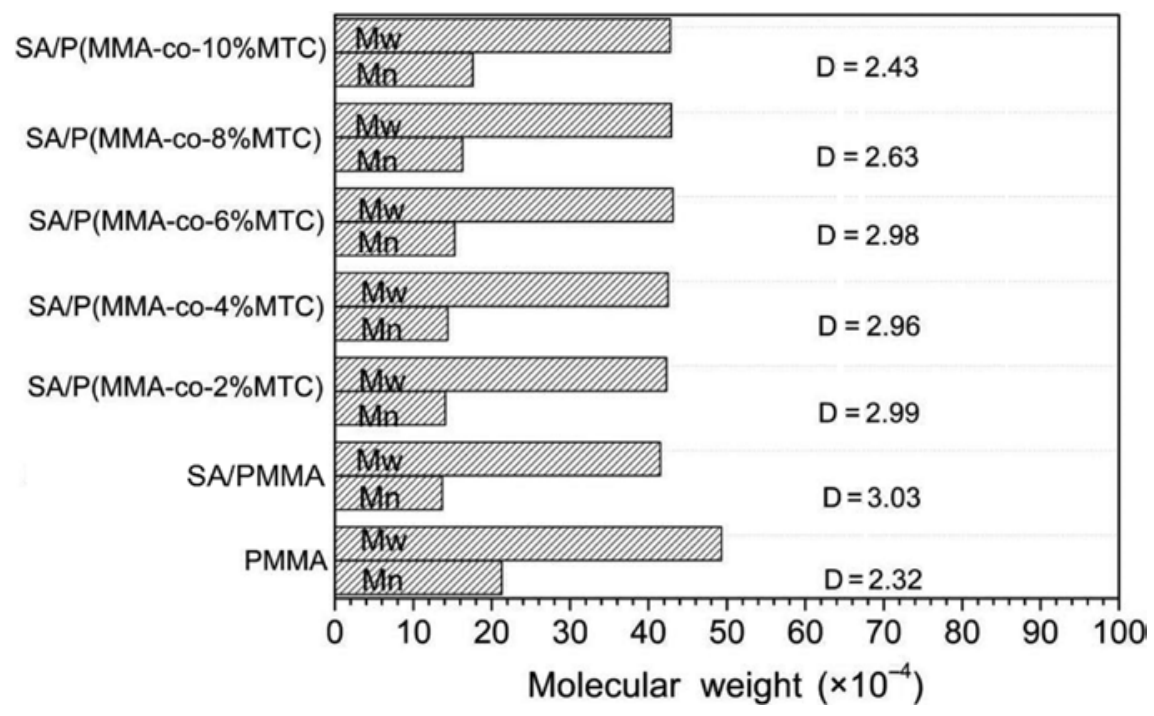

Figure 3: GPC results of samples.

could be absorbed on the SA skeleton's surface by electrostatic interaction.

Figure 3 shows gel permeation chromatography (GPC) results of PMMA, SA/PMMA, SA/P(MMA-co2\%MTC), SA/P(MMA-co-4\%MTC), SA/P(MMA-co-6\%MTC), SA/P(MMA-co-8\%MTC), and SA/P(MMA-co-10\%MTC).

The polydispersity index of PMMA is less than that of other samples, it suggested that the PMMA molecular weight distribution was narrow. The polydispersity index of SA/PMMA was larger than that of PMMA. The SA/PMMA molecular weight distribution was wide, and the size was not uniform. MMA could be adsorbed to the pores of SA, the MMA in the pores could be polymerized, and the MMA in other locations could not be polymerized. The PMMA radical polymerization could be hindered. Another reason was that when the PMMA to SA surface, the free radical active points disappeared, the SA acted as a polymerization inhibitor. The polydispersity index of SA/P(MMA-coMTC) is smaller than that of SA/PMMA, the reason is that the MTC has positive charges, SA has negative charges, and MTC would be absorbed to the SA skeleton surface with MMA.

With the increase of MTC in the reaction, MTC molecular chains excluded each other, and the movement space became larger; therefore, SA/P(MMA-co-MTC) molecular weight distribution is narrower than that of SA/PMMA. The number average ( $\mathrm{Mn})$ of PMMA is larger than that of other substances. The weight average (Mw) change of samples was not obvious and increased slightly. The MMA could be absorbed by the pores of SA, the MMA in the pores could be polymerized, and the other locations could not be polymerized. The SA could hinder PMMA radical polymerization, so the of SA/PMMA became small. With the increase in the proportion of MTC, the gradually increased. The reason was the MMA could be absorbed to the SA surface, which makes the PMMA concentration become large.

Figure 4 shows haze results of (A) SA, (B) PMMA, (C) P(MMA-co-MTC), (D) SA/PMMA, (E) PMMA-KH570SA, and (F) SA/P(MMA-co-MTC). The haze values of SA and SA/PMMA are larger than those of other samples, which are $5.735 \%$ and $5.264 \%$, respectively. The reason was that the SA and PMMA of SA/PMMA, through van der Waals forces, connected inorganic SA dispersed with organic PMMA. The two substances' compatibility was not good and the refractive index difference was large,

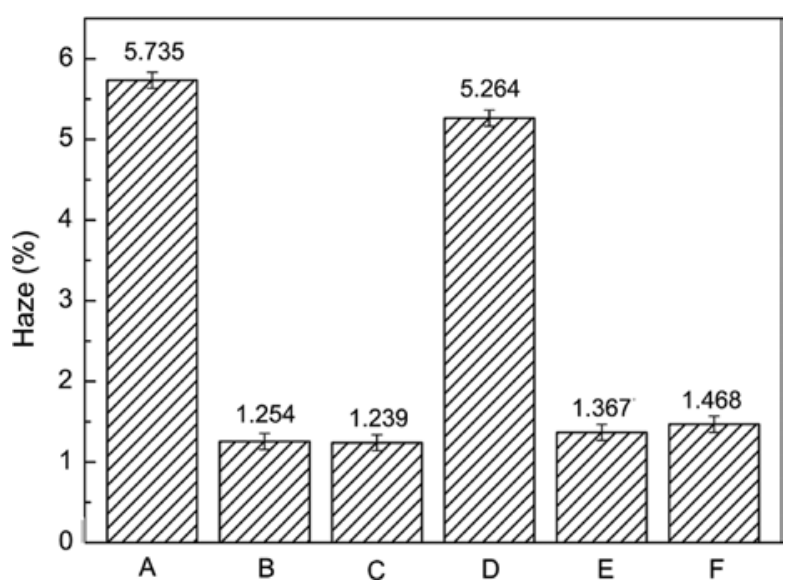

Figure 4: Haze results of samples (A) SA, (B) PMMA, (C) P(MMA-Co-MTC), (D) SA/PMMA, (E) PMMA-KH570-SA, and (F) SA/P(MMA-CO-MTC). 
in which the light through the material's different phase interfaces could cause scattering loss. Therefore, the SA/PMMA haze value is high. The PMMA-KH570-SA and SA/P(MMA-co-MTC) haze values are $1.367 \%$ and $1.468 \%$, respectively. PMMA-KH570-SA and SA/P(MMA-co-MTC) were linked by SA and PMMA through chemical bond and electrostatic interaction, respectively. The compatibility of SA and PMMA was improved through chemical method and electrostatic interaction, which could have phase interface; therefore, PMMA-KH570-SA and SA/P(MMA-co-MTC) would have a low haze value. The haze values of PMMA and P(MMA-co-MTC) were $1.254 \%$ and $1.239 \%$, respectively. The reason was that the MTC was added to PMMA, in which it could increase the gap between the molecular chains and decrease the haze value slightly.

Figure 5 shows visible-light transmittance of $\mathrm{P}(\mathrm{MMA}-$ co-MTC), SA, and SA/P(MMA-co-MTC). The light transmittance of P(MMA-co-MTC), SA, and SA/P(MMA-co-MTC) was at approximately 95\%, 90\% and 92\%, respectively. The light transmittance of P(MMA-co-MTC) is the best compared with that of SA/P(MMA-co-MTC) and SA in the visible-light range. SA/P(MMA-co-MTC) light transmittance is basically the same with the PMMA and it was slightly better than that of PMMA, SA light transmittance was bad. The phase interface of SA/P(MMA-co-MTC) was formed between the inorganic phase and the organic phase by electrostatic interaction. Because the phase interface affects the light transmittance, leading to the transmittance of SA/P(MMA-co-MTC), which is better than that of P(MMA-co-MTC).

The haze results of samples were analyzed in Figure 4, we have also done a refractive index experiment, through

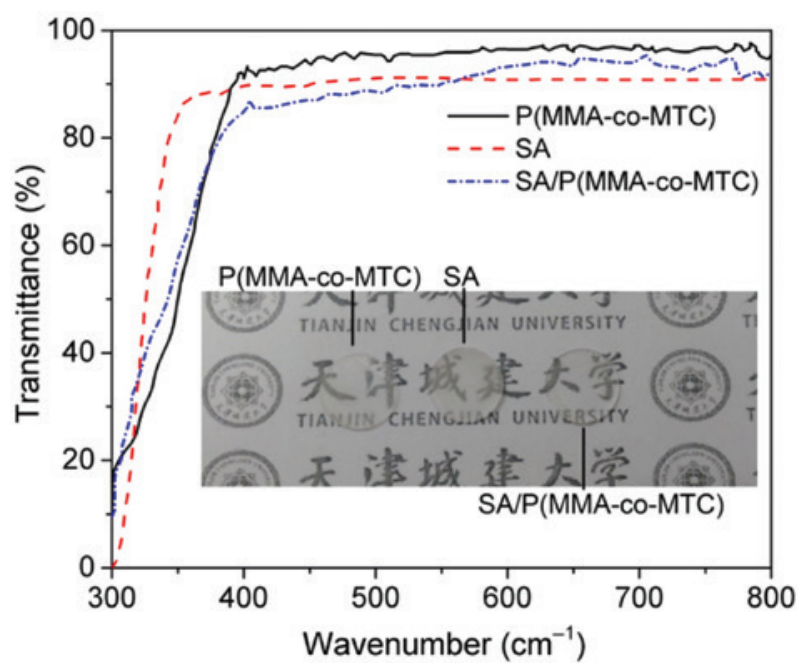

Figure 5: Transmittance of samples.

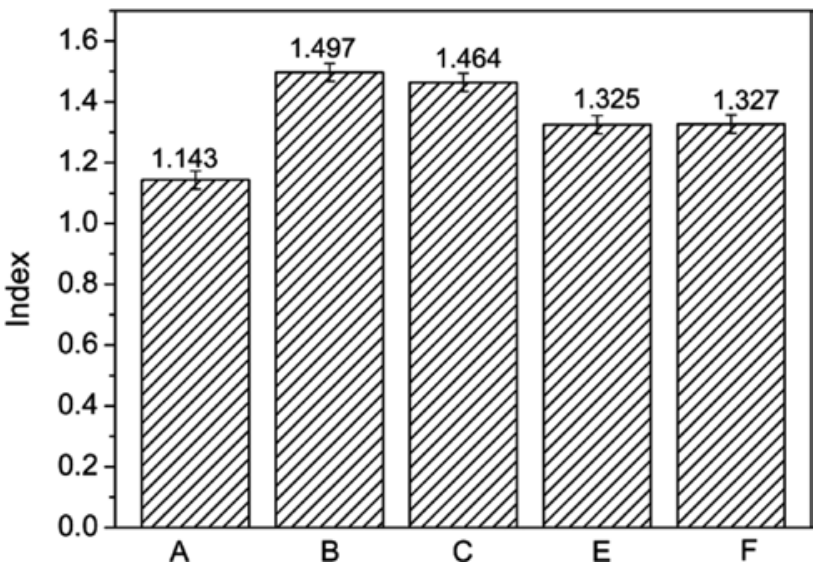

Figure 6: Refractive index results of samples: (A) SA, (B) PMMA, (C) P(MMA-co-MTC), (E) PMMA-KH570-SA, and (F) SA/P(MMA-co-MTC).

refractive index results, in which we could further analyze performance of samples clearly. Figure 6 shows refractive index results of (A) SA, (B) PMMA, (C) P(MMA-co-MTC), (E) PMMA-KH570-SA, and (F) SA/P(MMA-co-MTC).

The refractive index of $\mathrm{SA}$ is 1.143 , which is the minimum value among the samples. The refractive index of PMMA is 1.497. It is the highest one among the samples. The refractive indexes of the P(MMA-co-MTC), PMMA-KH570-SA, and SA/P(MMA-co-MTC) lie between those of SA and PMMA, which are 1.464, 1.325, and 1.327, respectively. SA and PMMA refractive index difference was large, MTC and KH570 could play a connection role; MTC and KH570 could be absorbed on the SA surface with MMA by electrostatic interaction and chemical bond, respectively, so the refractive indexes of P(MMA-co-MTC), PMMA-KH570-SA, and SA/P(MMA-co-MTC) are smaller than that of PMMA. It could be seen that the influence of the different phase interfaces on the refractive index of the composite was very low.

Figure 7 shows DSC results of (A) SA, (B) PMMA, (D) SA/PMMA, (G) SA/P(MMA-co-2\%MTC), (H) SA/P(MMAco-4\%MTC), (I) SA/P(MMA-co-6\%MTC), (J) SA/P(MMAco-8\%MTC), and (K) SA/P(MMA-co-10\%MTC). The glass transition temperature of samples can be obtained from curves and was summarized in Table 2 . As can be seen from Table 2, the Tg of PMMA and SA/PMMA are $113^{\circ} \mathrm{C}$ and $116^{\circ} \mathrm{C}$, respectively. The Tg of SA/PMMA is larger than that of PMMA; because some of the molecular chains were adsorbed into the pores, it could limit the movement of the molecular chains, groups, and others, and the glass transition temperature increases. The Tg of SA/PMMA and SA/P(MMA-co-2\%MTC) are $96^{\circ} \mathrm{C}$ and $99^{\circ} \mathrm{C}$, respectively. The MTC is the special functional cationic polymer monomer. It was presented in the copolymer, which could make the molecular chain have positive charges. The molecular 


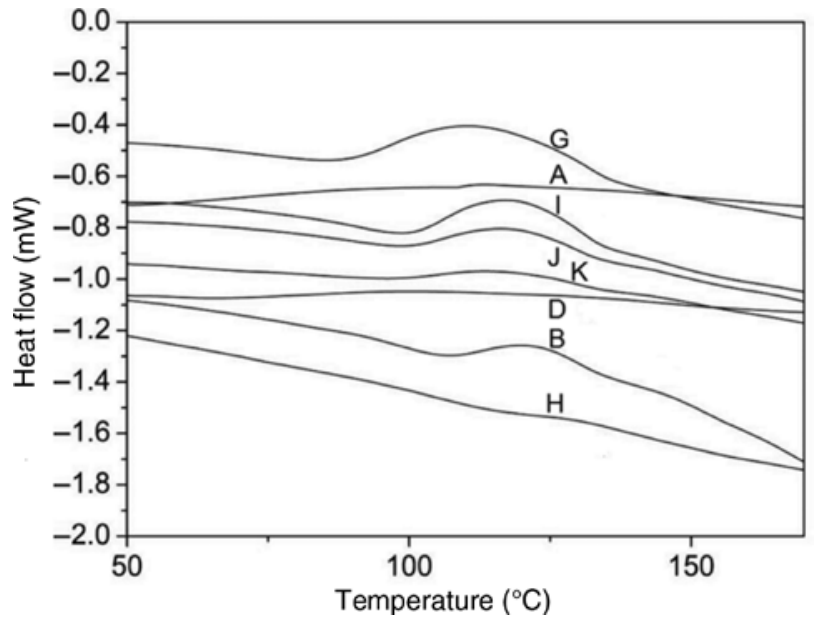

Figure 7: DSC results of samples: (A) SA, (B) PMMA, (D) SA/ PMMA, (G) SA/P(MMA-co-2\%MTC), (H) SA/P(MMA-co-4\%MTC), (I) SA/P(MMA-co-6\%MTC), (J) SA/P(MMA-co-8\%MTC), and (K) SA/P(MMA-Co-10\%MTC).

chain with the same charges excluded each other and became rigid molecules easily; therefore, the Tg increases with the increasing copolymerization ratio of MTC. The Tg of the SA/P(MMA-co-MTC) composite is slightly lower than that of the PMMA with the increasing copolymerization ratio of MTC. The P(MMA-co-MTC) increases the charges, the positive charges could cause a rejection between the molecular chains, and the gaps of the copolymer molecular chains increased. It could make the Tg of PMMA greater than that of composites in the copolymerization ratio of $0-10 \%$; therefore, the effect of the gap was larger than that of the rigid molecules on the Tg.

\section{Conclusion}

In this article, wet gel was prepared using the sol-gel method, SA was obtained by supercritical drying technology, and SA/P(MMA-co-MTC) composites were successfully prepared with the electrostatic interaction phase interface due to the negative charges in SA and positive charges in polymer. The haze, refractive index, transmittance, and other properties of the composites were studied. Through refractive index results, the refractive index of SA/P(MMA-co-MTC) was smaller than that of SA/PMMA. The reason was that the SA/P(MMA-co-MTC) composite had an electrostatic interaction phase interface, which could reduce the difference of the refractive index of SA and PMMA. FTIR spectra suggested that the SA/P(MMA-co-MTC) was present. DSC results suggested that the Tg of composites increased with the increasing copolymerization ratio of MTC. The MTC was the special functional cationic polymer monomer. It was presented in the copolymer, which could provide positive charges to the molecular chain. The polymer molecular chain excluded each other with the same charges and became rigid molecules easily. The visible-light transmittance results indicated that the transmittance of SA/P(MMAco-MTC) was better than that of SA. The reason was that the MTC could be absorbed on the SA surface, the interface between the inorganic phase and the organic phase was formed by electrostatic interaction. Based on these results, it can be confirmed that an electrostatic interaction phase interface between SA and polymer was formed.

Acknowledgments: This work was financially supported by the National Natural Science Foundation of China (Funder Id: 10.13039/501100001809, grants 51503141 and 51772202).

\section{References}

[1] Fricke J, Tillotson T. Thin Solid Films 1997, 297, 212-223.

[2] Deng ZS, Wang J, Wu AM, Shen J, Zhou B. J. Non Cryst. Solids 1998, 225, 101-104.

[3] He YL, Xie T. Appl. Therm. Eng. 2015, 81, 28-50.

[4] Xie T, He YL. Int. J. Heat Mass Transf. 2016, 95, 621-635.

[5] Wang XD, Sun D, Duan YY, Hu ZJ. J. Non Cryst. Solids 2013, 375, 31-39.

[6] Khan H, Amin M, Yasin M. J. Polym. Eng. 2016, 37, 671-680.

[7] Maleki H, Duraes L, Portugal A. J. Non Cryst. Solids 2014, 385, 55-74.

[8] Yuan B, Ding SQ, Wang DD, Wang G, Li HX. Mater. Lett. 2012, 75, 204-206.

[9] Yang X, Sun Y, Shi D, Liu J. Mater. Sci. Eng. 2011, 528, 4830-4836.

[10] Li Z, Cheng XD, He S, Shi XJ, Gong LL, Zhang HP. Compos. Part A 2016, 84, 316-325.

[11] Parmenter KE, Milstein F. J. Non Cryst. Solids 1998, 223, 179-189.

[12] Yang HX, Ye F, Liu Q, Gao Y. Mater. Des. 2015, 85, 438-443.

[13] Yuan B, Ding S, Wang D, Wang LH. Mater. Lett. 2012, 75, 204-206.

[14] Li J, Qin SH, He WT. J. Polym. Eng. 2015, 35, 565-574.

[15] Feng JZ, Zhang CR, Feng J, Jiang YG, Zhao N. ACS Appl. Mater. Interfaces 2011, 3, 4796-4803.

[16] Zhang G, Dass A, Rawashdeh AM, Thomas JS, Counsil JA, Sotiriouleventis C, Fabrizio EF, Ilhan F, Vassilaras P, Scheiman DA. J. Non Cryst. Solids 2004, 350, 152-164.

[17] Katti A, Shimpi N, Roy S, Lu H, Fabrizio EF, Dass A, Capadona LA, Leventis N. Chem. Mater. 2006, 18, 285-296.

[18] Leventis N, Sotiriouleventis C, Zhang GH, Rawashdeh AM. Nano Lett. 2002, 2, 957-960. 
[19] Chiu WM, Syu JS, Tsai PA. J. Polym. Eng. 2016, 36, 309-320.

[20] Scherer GW, Smith DM, Qiu X, Anderson JM. J. Non Cryst. Solids 1995, 186, 316-320.

[21] Scherer GW, Haereid S, Nilsen E, Einarsrud MA. J. Non Cryst. Solids 1996, 202, 42-52.

[22] Wang X, Jana SC. ACS Appl. Mater. Interfaces 2013, 5, 6423-6429.

[23] Meador MA, Fabrizio EF, Ilhan F, Dass A, Zhang GH, Vassilaras P, Johnston JC, Leventis N. Chem. Mater. 2005, 17, 1085-1098.
[24] Samantha N, Gilroy JB. Polym. Chem. 2017, 8, 5388-5395.

[25] Banerjee SJ, Ladmiral V, Debuigne A, Detrembleur C, Rahaman SM, Rinaldo P, Ameduri B. Macromol. Rapid Commun. 2017, 38, 1-7.

[26] Fieser ME, Sanford MJ, Mitchell LA. J. Am. Chem. Soc. 2017, 139, 15222-15231.

[27] Liu HL, Chung HY. Polym. Sci. 2017, 55, 3515-3528.

[28] Li HY, Song LM, Fu YQ, Wei YF, Li RY, Liu HL. Compos. Sci. Technol. 2017, 138, 169-178. 